\title{
Dynamics of topological defects in a spiral: a scenario for the spin-glass phase of cuprates
}

\author{
V. Juricic ${ }^{1}$, L. Benfatto ${ }^{1}$, A. O. Caldeira ${ }^{2}$, and C. Morais Smith ${ }^{1}$ \\ ${ }^{1}$ Département de Physique, Université de Fribourg, Pérolles, CH-1700 Fribourg, Switzerland. \\ ${ }^{2}$ Instituto de Física Gleb Wataghin, Universidade Estadual de Campinas, 13083-970, Campinas, SP, Brazil
}

\begin{abstract}
We propose that the dissipative dynamics of topological defects in a spiral state is responsible for the transport properties in the spin-glass phase of cuprates. Using the collective-coordinate method, we show that topological defects are coupled to a bath of magnetic excitations. By integrating out the bath degrees of freedom, we find that the dynamical properties of the topological defects are dissipative. The calculated damping matrix is related to the in-plane resistivity, which exhibits an anisotropy and linear temperature dependence in agreement with experimental data.
\end{abstract}

PACS numbers: 75.10.Nr, 74.25.Fy, 74.72.Dn

The discovery of four static incommensurate (IC) charge and spin peaks by neutron scattering experiments in Nd-doped $\mathrm{La}_{2-x} \mathrm{Sr}_{x} \mathrm{CuO}_{4}$ (LSCO) [1] confirmed the proposal that in these materials the holes form vertical/horizontal charge stripes 2], breaking the paradigm of homogeneous charge distribution. Moreover, the observation of IC dynamical spin correlations in superconducting LSCO $(x>0.05)$ at the same wave vectors as observed in the Nd-doped compounds [3] placed the stripe picture on a more solid footing and raised further questions concerning the coexistence of magnetism and superconductivity. Later, two IC elastic magnetic peaks have been observed within the spin-glass (SG) phase of LSCO $(0.02<x<0.05)$ [4], and their interpretation in terms of diagonal stripe formation appeared to be rather natural, given that the value of the magnetic IC follows the same linear dependence on doping as observed within the superconducting regime [4]. However, no charge order has ever been measured in the SG regime, and the picture of well-ordered stripes, very far apart from each other due to the very low doping concentration, seems quite improbable because disorder and frustration effects, which act to destabilize the stripes, are expected to dominate in this phase.

A different explanation of the two IC peaks in the SG phase is that these may arise from the formation of a spiral magnetic order which breaks the translational symmetry in the spin but not in the charge sector [5]. In this model, the randomly distributed holes act as frustration centers for the underlying anti-ferromagnetic (AF) background, generating a dipole moment. A fraction of these dipoles may order ferromagnetically, while the others may remain disordered. If the number of ordered dipoles increases linearly with the doping concentration, which would appear plausible, this model can describe the linear variation of the IC magnetic wave vector with doping [4], without invoking charge ordering [5].

Recently, transport properties along both diagonaldirections in the $\mathrm{Cu}$ lattice have been investigated in high-quality, detwinned LSCO samples, and a rather strong anisotropy was detected [6]. However, this experiment provides no definitive evidence in favor of the stripe picture, because, as we will show, a similar anisotropic behavior is expected for transport parallel or perpendicular to the spiral axis.

In this Letter we investigate the formation of topological defects in the spiral state that couple to excitations of the magnetic environment and diffuse across the system. We assume that the charge carriers are attached to these defects and therefore the description of defect dynamics corresponds ultimately to the electrical transport properties of cuprates in the SG phase. We evaluate the damping matrix, which is related to the mobility of the topological defect, and show that its behavior at high temperatures follows the experimentally observed temperature $(T)$ dependence of the in-plane resistivity. In addition, we account for the anisotropy in the spin-wave velocity in the directions parallel and perpendicular to the spiral axis, and find that, in agreement with experiments, it leads to an anisotropy in the resistivity.

The magnetic properties of charge transfer insulators, such as undoped $\mathrm{La}_{2} \mathrm{CuO}_{4}$, are usually studied within the quantum non-linear sigma model $(\mathrm{NL} \sigma \mathrm{M})$, which can correctly represent the long wavelength modes of the Heisenberg model. This continuum model describes slow fluctuations of the locally well defined staggered magnetization $\mathbf{n}$ (with $\mathbf{n}^{2}=1$ ). When holes are added to the system, the magnetism becomes more complex because those act simultaneously as a source of disorder and dipolarlike frustration. The dipolar frustration can be described via a coupling of the dipoles to the gradient of the order parameter $\mathbf{n}$ of the NL $\sigma \mathrm{M}$. By accounting for the random distribution of dipolar centers, the resulting Hamiltonian favors the formation of a spiral phase, with a non-zero average twist $\partial_{\mu} \mathbf{n}$ of the $\mathrm{AF}$ order parameter and a concomitant alignment of some of the dipoles [5].

The spiral ground-state breaks the $\mathrm{O}(3)$ spin symmetry completely. Its order parameter, which belongs to the $\mathrm{SO}(3)$ group, is given in terms of an orthonormal basis $\mathbf{n}_{k}$, with $k=1,2,3$ and $n_{k}^{a} n_{q}^{a}=\delta_{k q}$ [7]. 
The latter can be related to an element $g$ of $\mathrm{SU}(2)$ through $n_{k}^{a}=(1 / 2) \operatorname{tr}\left(\sigma^{a} g \sigma^{k} g^{-1}\right)$, where $\sigma^{a}$ are Pauli matrices. It is also convenient to introduce the fields $A_{\mu}^{a}=(1 / 2 i) \operatorname{tr}\left(\sigma^{a} g^{-1} \partial_{\mu} g\right)$, which are related to the first derivatives of $\mathbf{n}_{k}$ through $\partial_{\mu} n_{k}^{a}=2 \epsilon_{i j k} A_{\mu}^{i} n_{j}^{a}$ [8]. Here $\mu$ stands for one-time and two-spatial coordinates. The Lagrangian describing the spiral state is given by

$$
L=\mathcal{N} \int d x_{\|} d x_{\perp}\left(\mathbf{A}_{0}^{2}-c_{\perp}^{2} \mathbf{A}_{\perp}^{2}-c_{\|}^{2} \mathbf{A}_{\|}^{2}\right),
$$

where $\mathcal{N}=2 J S^{2} / c_{\|} c_{\perp}, J$ is the AF exchange and $c_{\perp}, c_{\|}$ are the spin wave velocities perpendicular and parallel to the spiral axis, with $\left(c_{\|} / c_{\perp}\right)^{2}=\cos (Q a)[\underline{5}$ ]. Here $a$ is the lattice constant and $Q$ is the IC magnetic wave vector observed by neutron scattering. Lagrangian (1) may be mapped to an isotropic form by introducing the coordinates $\mathbf{r}=(x, y)$ with $x=\sqrt{c_{\|} / c_{\perp}} x_{\perp}, y=\sqrt{c_{\perp} / c_{\|}} x_{\|}$. We then find $L=\mathcal{N} \int d^{2} r\left(\mathbf{A}_{0}^{2}-c^{2} \mathbf{A}_{\alpha}^{2}\right)$, where $\alpha=x, y$ and $c=\sqrt{c_{\perp} c_{\|}}$is the isotropic spin-wave velocity. This rescaling leads to an anisotropic elementary cell, which will be neglected in what follows. Further comments on the anisotropy effects are included in the conclusions.

An appropriate treatment of the spiral state has to take into account the existence of topological defects. In principle, in $(2+1)$ space-time dimensions two types of static topological defects can exist: vortices and skyrmions. Skyrmionlike excitations are classified according to the second homotopy group of the order parameter space, which in our case is trivial, $\pi_{2}(S O(3))=0$. Thus, there are no skyrmions in the spiral state and the only possible defects are $Z_{2}$-vortices, given by the first homotopy group $\pi_{1}(S O(3))=Z_{2}[\underline{9}$. These defects originate from the chiral degeneracy of the spiral, which can rotate clock- or counter-clock-wise [10]. Their description is provided by the elements $g=g_{s}(\Psi) g_{\varepsilon}(\vec{\varepsilon})$ of the $\mathrm{SU}(2)$ group, with $g_{s}=\exp \left(i m^{a} \sigma^{a} \Psi / 2\right)$ and $g_{\varepsilon}=\exp \left(i \sigma^{a} \varepsilon^{a} / 2\right)$. The parameters $\mathbf{m} \Psi$ are related to the local spin, where $\mathbf{m}$ is a constant unit vector and $\Psi$ is a scalar field 11. Our aim is to determine the topological defect solutions $\Psi_{2 v}$ of the saddle point equations of the spiral, as well as the vectorial field $\vec{\varepsilon}$ describing fluctuations around this solution. In terms of these fields, the Lagrangian becomes

$$
\begin{aligned}
L & =\mathcal{N} \int d^{2} r\left(L_{0}+L_{1}\right), \quad L_{0}=\frac{1}{4}\left(\partial_{\mu} \Psi\right)^{2} \\
L_{1} & =\frac{1}{4}\left(\partial_{\mu} \vec{\varepsilon}\right)^{2}+\frac{1}{2}\left\{\mathbf{m} \cdot\left[\partial_{\mu} \vec{\varepsilon}+\frac{1}{2}\left(\partial_{\mu} \vec{\varepsilon} \times \vec{\varepsilon}\right)\right]\right\} \partial_{\mu} \Psi,
\end{aligned}
$$

with $\partial_{\mu} A \partial_{\mu} B=\partial_{t} A \partial_{t} B-c^{2} \nabla A \nabla B$. This Lagrangian can be treated perturbatively because $|\vec{\varepsilon}|$ is small. We begin by considering the static limit of $L_{0}$, where the corresponding equation of motion becomes the Laplace equation $\nabla^{2} \Psi(\mathbf{r})=0$. A nontrivial solution $\Psi_{1 v}(\mathbf{r}, \mathbf{R})=$ $\arctan (y-Y) /(x-X)$ has the form of a vortex centered at $\mathbf{R}=(X, Y)$, and its energy $E \propto \ln L / a$ diverges with the system size $L$. Unbound, free topological defects cannot, therefore, exist at low temperatures. However, the twovortices solution $\Psi_{2 v}=\Psi_{1 v}\left(\mathbf{r}, \mathbf{R}_{1}\right)-\Psi_{1 v}\left(\mathbf{r}, \mathbf{R}_{2}\right)$, which describes a bound pair of topological defects (vortex and antivortex), centered respectively at $\mathbf{R}_{1}$ and $\mathbf{R}_{2}$, has a finite energy $E\left(\Psi_{2 v}\right) \propto \ln (d / a)$, where $d=\left|\mathbf{R}_{1}-\mathbf{R}_{2}\right|$ is the modulus of the pair relative coordinate, which we take as a constant. Up to an irrelevant constant, the twovortices solution can be expressed in terms of the center of mass $\mathbf{R}=\left(\mathbf{R}_{1}+\mathbf{R}_{2}\right) / 2$ and relative coordinate $\mathbf{d}$, yielding $\Psi_{2 v}(\mathbf{r})=\arctan \left\{[(\mathbf{r}-\mathbf{R}) \times \mathbf{d}]_{z} /\left[(\mathbf{r}-\mathbf{R})^{2}-d^{2} / 4\right]\right\}$.

We will apply the collective coordinate method [12] to study the dynamics of a bound pair of topological defects in a spiral. The first task is to determine the dynamics of the fluctuations around the defect. By evaluating $L_{1}\left(\Psi_{2 v}\right)$ and expressing the vectorial field in terms of polar coordinates, $\vec{\varepsilon}=\left(\bar{\varepsilon} \cos \theta, \bar{\varepsilon} \sin \theta, \varepsilon^{z}\right)$, we find that the $\varepsilon^{z}$ component is free and that $\theta=\Psi_{2 v} / 2$. The remaining equation of motion then reads $\left\{\partial_{t}^{2}-c^{2}\left[\nabla^{2}+V(\mathbf{r})\right]\right\} \bar{\varepsilon}=$ 0 , with $V(\mathbf{r})=\left(\nabla \Psi_{2 v}\right)^{2} / 4$. Its solution has the form $\bar{\varepsilon}(\mathbf{r}, t)=\sum_{n m} q_{n m}(t) \eta_{n m}(\mathbf{r})$, where $\eta_{n m}$ are the eigenfunctions of the operator

$$
-c^{2}\left[\nabla^{2}+V(\mathbf{r})\right] \eta_{n m}=\omega_{n m}^{2} \eta_{n m} .
$$

The solutions of Eq. (3) have the form $\eta_{n m}=$ $\sqrt{k_{n m} / 8 L}\left[H_{n}^{(1)}\left(k_{n m} r\right)+e^{-2 i \delta_{n}} H_{n}^{(2)}\left(k_{n m} r\right)\right] e^{i n \vartheta}$, where $H_{n}^{(1,2)}$ are Hankel functions of the first and second kinds, $\delta_{n}$ represents a phase shift for the mode $n$, and $\vartheta$ is a polar angle. The excitation with frequency $\omega_{n m}$ has the dispersion $\omega_{n m}=c\left|\mathbf{k}_{n m}\right|$. Equation (3) admits zerofrequency modes associated with the continuous translational symmetry of the model. A consistent treatment of them requires the use of collective coordinates. Thus, the center of the pair $\mathbf{R}$ is promoted to a dynamical variable $\mathbf{R}(t)$, namely $\Psi(\mathbf{r}) \rightarrow \Psi(\mathbf{r}-\mathbf{R}(t))$ and $\bar{\varepsilon}(\mathbf{r}, t) \rightarrow \bar{\varepsilon}(\mathbf{r}-\mathbf{R}(t), t)=\sum_{n m} q_{n m}(t) \eta_{n m}(\mathbf{r}-\mathbf{R}(t))$.

A lengthy but straightforward procedure [13] leads to the effective Hamiltonian (in units of $2 J S^{2} / c^{2}$ ) describing the pair of topological defects interacting with the bath of magnons,

$$
H=\frac{1}{2 M}\left(\mathbf{P}-\mathbf{P}_{E}\right)^{2}+\frac{1}{2} \sum_{n m}\left(p_{n m}^{2}+\omega_{n m}^{2} q_{n m}^{2}\right),
$$

where $M=\int d^{2} r\left(\nabla \Psi_{2 v}\right)^{2}$ is the mass of the topological defect, $\mathbf{P}$ and $p_{n m}$ are respectively the momentum conjugate to $\mathbf{R}$ and to the coordinate $q_{n m}$ of the excitation with frequency $\omega_{n m}$, and $\mathbf{P}_{E}=\sum_{n m, k l} p_{n m} \mathbf{G}_{n m, k l} q_{k l}$. The constants $\mathbf{G}_{n m, k l}$ are related to the eigenfunctions $\eta$ by $\mathbf{G}_{n m, k l}=\int d^{2} r \eta_{k l} \nabla \eta_{n m}$. The classical Hamiltonian (4) is quantized by introducing the usual creation and annihilation operators $a_{n m}^{\dagger}$ and $a_{n m}$. Restricting the analysis to the case of elastic scattering and retaining only the terms at quadratic order in $a_{n m}^{\dagger}, a_{n m}$ we obtain

$$
\hat{H}=\frac{\hat{\mathbf{P}^{2}}}{2 M}-\frac{\hbar \hat{\mathbf{P}}}{M} \sum_{n m, k l} \mathbf{D}_{n m, k l} a_{n m} a_{k l}^{\dagger}+\hat{H}_{b},
$$


where $\hat{H}_{b}=\sum_{n m} \hbar \omega_{n m} a_{n m}^{\dagger} a_{n m}$ and $\mathbf{D}_{n m, k l}=$ $\mathbf{G}_{n m, k l}\left(\omega_{n m}+\omega_{k l}\right) / 2 i \sqrt{\omega_{n m} \omega_{k l}}$.

Our aim is to investigate, in the low-energy sector, the effective dynamics of the vortex-pair in the presence of the excitation bath. We compute the reduced density matrix of the pair by integrating out the degrees of freedom of the bath of magnons, for which we employ the Feynman-Vernon path-integral formalism. The evolution of the density matrix for the full system is described by $\hat{\rho}(t)=\exp (-i \hat{H} t / \hbar) \hat{\rho}(0) \exp (i \hat{H} t / \hbar)$. For the sake of simplicity, we use the factorisable initial condition $\hat{\rho}(0)=\hat{\rho}_{v}(0) \hat{\rho}_{b}(0)$, where $\rho_{v}$ and $\hat{\rho}_{b}$ represent, respectively, the initial vortex-pair and bath density matrices. This condition implies that the pair of topological defects and the excitations do not interact at $t=0$. The bath degrees of freedom are supposed to be initially in thermal equilibrium, $\hat{\rho}_{b}(0)=e^{-\beta \hat{H}_{b}} / \operatorname{tr}\left[e^{-\beta \hat{H}_{b}}\right]$, where $\beta \equiv \hbar / k_{B} T$. After evaluating the trace over these, we obtain the reduced density-matrix operator for the vortexpair

$$
\hat{\tilde{\rho}}(\mathbf{x}, \mathbf{y}, t)=\iint d \mathbf{x}^{\prime} d \mathbf{y}^{\prime} J\left(\mathbf{x}, \mathbf{y}, t ; \mathbf{x}^{\prime}, \mathbf{y}^{\prime}, 0\right) \hat{\rho}_{v}\left(\mathbf{x}^{\prime}, \mathbf{y}^{\prime}, 0\right),
$$

$$
\begin{aligned}
\Phi & =2 \sum_{\mu, \nu=1}^{2} \int_{0}^{t} d t^{\prime} \int_{0}^{t^{\prime}} d t^{\prime \prime} \epsilon^{\mu \nu}\left(t^{\prime}-t^{\prime \prime}\right) \dot{u}^{\mu}\left(t^{\prime}\right) \dot{v}^{\nu}\left(t^{\prime \prime}\right), \\
\epsilon^{\mu \nu}(t) & =\sum_{n m, k l \neq 0} N_{n m} D_{n m, k l}^{\mu} D_{n m, k l}^{\nu} \sin \left(\omega_{n m}-\omega_{k l}\right) t,
\end{aligned}
$$

The boson occupation number is $N_{n m}=\left(e^{\beta \omega_{n m}}-1\right)^{-1}$. The effective action that describes the motion of the vortex pair in the presence of excitations may then be obtained by inserting Eq. (77) into the superpropagator (6). We find that $S_{\text {eff }}=S_{0}[\mathbf{x}]-S_{0}[\mathbf{y}]+\hbar \Phi$ and the corresponding equations of motion indicate that the dynamics of the defects is damped,

$$
\begin{aligned}
& \ddot{v}^{\mu}(\tau)+\sum_{\nu} \int_{0}^{\tau} d t^{\prime} \gamma^{\mu \nu}\left(\tau-t^{\prime}\right) \dot{v}^{\nu}\left(t^{\prime}\right)=0, \\
& \ddot{u}^{\mu}(\tau)-\sum_{\nu} \int_{\tau}^{t} d t^{\prime} \gamma^{\mu \nu}\left(t^{\prime}-\tau\right) \dot{u}^{\nu}\left(t^{\prime}\right)=0,
\end{aligned}
$$

where the matrix $\gamma^{\mu \nu}(t)=(2 \hbar / M) \dot{\epsilon}^{\mu \nu}(t)$ is a generalization of the damping coefficient. The decaying part $\exp [\tilde{\Phi}]$ of the influence functional is related to the diffusive properties of the defect, with the diffusion matrix $\mathcal{D}^{\mu \nu}(t)=\hbar \ddot{\tilde{\epsilon}}^{\mu \nu}(t)$. The evaluation of the diffusion is analogous to that of the damping matrix, and at low $T$ the two parameters are related by the fluctuationdissipation theorem. Here we will concentrate on $\gamma$ only. We first introduce the scattering function $S^{\mu \nu}\left(\omega, \omega^{\prime}\right)=$ where the super-propagator $J$ has the form

$$
J=\int_{\mathbf{x}^{\prime}}^{\mathbf{x}} \mathcal{D} \mathbf{x} \int_{\mathbf{y}^{\prime}}^{\mathbf{y}} \mathcal{D} \mathbf{y} e^{\frac{i}{\hbar}\left[S_{0}[\mathbf{x}]-S_{0}[\mathbf{y}]\right]} \mathcal{F}[\mathbf{x}, \mathbf{y}]
$$

$S_{0}[\mathbf{x}]=\int_{0}^{t} d t^{\prime}(M / 2) \dot{\mathbf{x}}^{2}$ is the action associated with the free motion of the vortices and $\mathcal{F}[\mathbf{x}, \mathbf{y}]$ is the influence functional, which describes the effect of the excitations on the dynamics of the topological defect pair.

Defining the center of mass and relative coordinates $\mathbf{v}=(\mathbf{x}+\mathbf{y}) / 2, \mathbf{u}=\mathbf{x}-\mathbf{y}$ and using the Born approximation, one finds, after a lengthy calculation [13], that

$$
\mathcal{F}[\mathbf{x}, \mathbf{y}]=\exp [i \Phi] \exp [\tilde{\Phi}]
$$

where

$$
\begin{gathered}
\tilde{\Phi}=\sum_{\mu, \nu=1}^{2} \int_{0}^{t} d t^{\prime} \int_{0}^{t^{\prime}} d t^{\prime \prime} \tilde{\epsilon}^{\mu \nu}\left(t^{\prime}-t^{\prime \prime}\right) \dot{u}^{\mu}\left(t^{\prime}\right) \dot{u}^{\nu}\left(t^{\prime \prime}\right), \\
\tilde{\epsilon}^{\mu \nu}(t)=\sum_{n m, k l \neq 0} N_{n m} D_{n m, k l}^{\mu} D_{n m, k l}^{\nu} \cos \left(\omega_{n m}-\omega_{k l}\right) t .
\end{gathered}
$$

$\sum G_{n m, k l}^{\mu} G_{n m, k l}^{\nu} \delta\left(\omega-\omega_{n m}\right) \delta\left(\omega^{\prime}-\omega_{k l}\right)$, which allows the description of the problem in terms of continuous frequencies. Because the model we study is isotropic, the damping matrix is diagonal $\gamma^{\mu \nu}(t)=\gamma(t) \delta^{\mu \nu}$, and $\gamma(t)$ is obtained directly from the first derivative $\dot{\epsilon}(t)$. After introducing the substitution $\xi=\left(\omega+\omega^{\prime}\right) / 2, \zeta=\omega-\omega^{\prime}$, and considering only processes for which $\zeta<<1$, we find that $\gamma(t)=\bar{\gamma}(T) \delta(t)$ with

$$
\begin{aligned}
\bar{\gamma}(T) & =-\frac{2 \pi \hbar}{M} \int_{0}^{\infty} d \xi \varphi(\xi) \frac{\partial N(\xi)}{\partial \xi} \\
\varphi(\xi) & =\frac{\xi^{2}}{2 c^{2}} \sum_{n=1}^{\infty} \sin ^{2}\left(\delta_{n+1}-\delta_{n}\right)
\end{aligned}
$$

The phase shifts $\delta_{n}$ of the eigenfunctions $\eta_{n m}$ can be calculated using the Fredholm method [12, 13]. Their evaluation requires the computation of the matrix elements $\mathcal{A}_{n}=\langle n|V| n\rangle$, because $\delta_{n}=\arctan \left[\pi \mathcal{A}_{n} /(1+\right.$ $\left.\mathcal{A}_{n}\right)$ ]. Here $|n\rangle$ are the eigenstates of the unperturbed operator in Eq. (3). In the limit of a small vortexantivortex separation, $d \ll r$, the potential reads $V(r)=$ 
$d^{2} /\left[4\left(r^{2}+d^{2} / 4\right)^{2}\right]$. One then obtains

$$
\mathcal{A}_{n}(\xi)=-\pi \xi \partial_{\xi}\left[I_{n}(\xi d / 2 c) K_{n}(\xi d / 2 c)\right],
$$

where $I_{n}, K_{n}$ are the modified Bessel functions of the first and the second kinds, respectively. Using the Drude model and assuming that the charge carriers are attached to the defects, we can relate $\bar{\gamma}$ to the inverse of the mobility, $\mu^{-1}=M \bar{\gamma} / e$. We then find

$$
\begin{aligned}
\mu^{-1} & =\frac{\pi \hbar \beta}{e c^{2}} \sum_{n=1}^{\infty} \int_{0}^{\infty} d \xi \frac{\xi^{2} e^{\beta \xi}}{\left(e^{\beta \xi}-1\right)^{2}} \frac{\mathcal{B}_{n}^{2}(\xi)}{\left[1+\mathcal{B}_{n}^{2}(\xi)\right]} \\
\mathcal{B}_{n} & =\frac{\pi\left(\mathcal{A}_{n+1}-\mathcal{A}_{n}\right)}{1+\mathcal{A}_{n}+\mathcal{A}_{n+1}+\left(1+\pi^{2}\right) \mathcal{A}_{n} \mathcal{A}_{n+1}}
\end{aligned}
$$

This expression leads to a vanishing $\mu^{-1}$ as $T \rightarrow 0$ because in this limit there are no magnons to scatter the topological defects and the latter behave as free particles. However, this limit will never be reached in systems like LSCO because at $T_{f} \sim 30 \mathrm{~K}$ the dipoles freeze and the charge becomes localized, leading to an upturn of the resistivity. At high $T$ we obtain

$$
\mu^{-1}(T)=\frac{\pi k_{B} T}{e c^{2}} \sum_{n} \int_{0}^{\infty} d \xi \frac{\mathcal{B}_{n}^{2}(\xi)}{1+\mathcal{B}_{n}^{2}(\xi)}
$$

which indicates that the resistivity $\rho=\left(\mu n_{h} e\right)^{-1}$ varies linearly with $T$, as observed experimentally $[\underline{6}$, 14]. Here $n_{h}$ denotes the charge carriers concentration. This behavior is expected to hold for $T<T_{K T}$, where $T_{K T}$ is the Kosterlitz-Thouless temperature, at which the vortex anti-vortex pairs will eventually unbind. An attempt to roughly estimate $T_{K T}$ has provided $T_{K T} \sim J S^{2}$ [ 5 . Using that $J \sim 0.1 \mathrm{eV}$ and $S=1 / 2$, we find $T_{K T} \sim 300 \mathrm{~K}$. Despite of the crudeness of the estimates, this result compares quite well with the experimental data [14], which shows deviations from linearity at $T \sim 400 \mathrm{~K}$. Given that $T_{K T}$ represents the temperature at which few pairs start to unbind, it is not surprising that the linear behavior can hold up to higher T.

The order of magnitude of the resistivity can also be promptly evaluated from our calculations. Although the exact microscopic values of the parameters $d$ and $c$ are not available, we nevertheless provide an estimate of these. Taking $\hbar c / a k_{B} \sim T_{K T}$ and $d \sim a$, we find $\mu^{-1} \sim 0.1 \mathrm{Vs} / \mathrm{cm}^{2}$ for $T=200 \mathrm{~K}$, in good agreement with Fig. 2 of Ref. [6].

In order to provide also a comparison with the experimental data concerning the anisotropy in dc transport, we should include the spin-wave anisotropy in the spiral state. The analytical calculations which led to Eqs. (9) - (11) cannot be performed for an anisotropic magnon bath. Nevertheless, we provide a rough estimate of this effect by considering the dependence of the inverse mobility in Eq. (11) on the spin-wave velocity $c$. Recalling that $\mathcal{B}_{n}$ is a function of $\xi d / 2 c$, we find that $\mu^{-1} \propto c^{-1}$.
One could then expect that in the original anisotropic system $\mu_{\perp}^{-1} \propto c_{\perp}^{-1}$ and $\mu_{\|}^{-1} \propto c_{\|}^{-1}$. This result can also be understood on physical grounds: since dissipation is provided by the excitations of the magnon bath, spinwaves with higher velocity are less effective in scattering the defects. As a consequence, the resistivity in the spiral direction is larger than that in the direction perpendicular to it, $\gamma_{\perp} / \gamma_{\|}=\rho_{\perp} / \rho_{\|}=c_{\|} / c_{\perp}=\sqrt{\cos (Q a)}$. For $x=0.04$, we find $\sqrt{\cos (Q a)}=0.98$, in agreement with transport measurements by Ando et al. [6], which found that for $100 \mathrm{~K}<T<200 \mathrm{~K}$ the resistivity along the $a$-axis in LSCO is slightly smaller than along the $b$ axis (in orthorhombic coordinates). We note that the IC peaks observed in neutron scattering correspond to the $b$-direction, which coincides with the spiral axis because the breaking of translational symmetry within the spiral picture has its origin in the spiral chirality (which can rotate clock- or counter-clockwise). Thus, $\rho_{\perp} \equiv \rho_{a}, \rho_{\|} \equiv \rho_{b}$ and $\rho_{a}<\rho_{b}$, as experimentally observed. This result indicates that the anisotropy measured in the SG phase does not provide evidence for the existence of diagonal stripes, but instead, is also the result expected within a more realistic, albeit more complex, model which does not need to appeal to charge order at such small doping concentrations.

In conclusion, we propose a description of the transport properties in the SG phase of cuprates based on the dissipative dynamics of topological defects in a spiral state. Using the collective-coordinate method, we derive the effective action of the topological defects, which are coupled to a bath of magnons. The scattering of magnons by the potential provided by the topological defects leads to a dissipative motion for these defects. Under the assumption that the holes are attached to the defects, the corresponding damping matrix is calculated, and is related to the in-plane resistivity. Its $T$ dependence and anisotropic behavior are in agreement with the available experimental data, indicating that further investigations are required to distinguish between spiral spin states and diagonal stripes in the SG phase of cuprates.

We acknowledge fruitful discussions with N. Hasselmann, A. H. Castro Neto, V. Gritsev, and A. Villares Ferrer. One of us (AOC) wishes to thank Fundação de Amparo à Pesquisa no Estado de São Paulo (FAPESP) and Conselho Nacional de Desenvolvimento Científico e Tecnológico $(\mathrm{CNPq})$ for their financial aid. This work was mainly supported by the Swiss National Foundation for Scientific Research under grant No. 620-62868.00.

[1] J. M. Tranquada et al., Nature (London) 375, 561 (1995).

[2] J. Zaanen and O. Gunnarsson, Phys. Rev. B 40, 7391 (1989).

[3] K. Yamada et al., Phys. Rev. B 57, 6165 (1998). 
[4] S. Wakimoto et al., Phys. Rev. B 60, R769 (1999); 61, 3699 (2000); M. Matsuda et al., ibid. 62, 9148 (2000); M. Fujita et al., ibid. 61, 3699 (2000); 65, 064505 (2002).

[5] N. Hasselmann, A. H. Castro Neto, and C. Morais Smith, Europhys. Lett. 56, 870 (2001); Phys. Rev. B 69, 014424 (2004).

[6] Y. Ando et al., Phys. Rev. Lett. 87, 017001 (2001); ibid. 88, 137005 (2002).

[7] W. Apel et al., Zeit. Phys. B 86, 139 (1992).

[8] A. M. Polyakov, Phys. Lett. B 59 , 79 (1975).

[9] T. Dombre and N. Read, Phys. Rev. B 39, 6797 (1989).
[10] H. Kawamura, J. Phys. Condens. Matter 10, 4707 (1998).

[11] M. Wintel, H. U. Everts, and W. Apel, Europhys. Lett. 25, 711 (1994); Phys. Rev. B 52, 13480 (1995).

[12] A. H. Castro Neto and A. O. Caldeira, Phys. Rev. Lett. 67, 1960 (1991); Phys. Rev. B 46, 8858 (1992); Phys. Rev. E 48, 4037 (1993); A. V. Ferrer and A. O. Caldeira, Phys. Rev. B 61, 2755 (2000).

[13] V. Juricic et al. (unpublished).

[14] H. Takagi et al., Phys. Rev. Lett. 69, 2975 (1992). 